\title{
Surgical Treatment of Small Intestinal Neuroendocrine Tumors G1/G2
}

\author{
Andreas Selberherr ${ }^{a} \quad$ Martin B. Niederle ${ }^{b} \quad$ Bruno Niederle $^{c}$ \\ aDivision of General Surgery, Department of Surgery, Medical University of Vienna, Vienna, Austria; \\ ${ }^{b}$ Department of General Anesthesia, General Intensive Care and Pain Management, Medical University of Vienna, Vienna, Austria; \\ ${ }^{\mathrm{c}}$ Department of Surgery, Franziskus Spital, Vienna, Austria
}

\section{Keywords}

Neuroendocrine tumor - Neuroendocrine neoplasia .

Small intestine $\cdot$ Jejunum $\cdot$ lleum

\section{Summary}

Although the majority of neuroendocrine tumors of the small intestine (siNETs) classified as low-grade G1 or G2 show slow local growth, they are frequently diagnosed at an advanced stage of metastatic disease. The surgical treatment is curative in stages I-III or palliative in stage IV in an attempt to avoid local complications of bowel obstruction and ischemia of the small bowel by unremoved lymph node metastases. Individualized surgical procedures performed by experienced surgeons considering tumor multifocality and the primary extent of lymph node metastases along the mesenteric vessels are recommended to remove as much tumor volume as possible, while avoiding major complications intraoperatively and small bowel syndrome postoperatively.

(C) 2017 S. Karger GmbH, Freiburg

\section{Introduction}

Neuroendocrine tumors (NETs) of the small intestine (siNETs) were first described by Otto Lubarsch in 1888 [1].

In 1907, the German pathologist Siegfried Oberndorfer referred to these tumors as characterized by the same morphology as 'carcinoids' [2].

Compared to 'carcinoma', the term 'carcinoid' describes a less aggressive malignant tumor entity. Due to the high level of heterogeneity seen within tumor behavior, ranging from benign to uncer- tain to malignant, the term 'carcinoid' failed to adequately characterize such tumors. Therefore, the term 'carcinoid' was replaced by the term 'neuroendocrine neoplasia' in general [3].

\section{Incidence}

By definition, 1.49\% of all malignant tumors of the digestive tract documented in the study period arose from neuroendocrine cells [4]. siNETs comprise $0.56 \%$ of all reported malignancies. In a prospective study, the annual incidence rate of neuroendocrine neoplasia of the gastroenteropancreatic tract was 2.51 per 100,000 inhabitants for men and 2.36 per 100,000 for women. The small intestine was the main site in $15 \%$ [4]. The incidence of malignant gastrointestinal NETs was 0.81 per 100,000 inhabitants and the incidence of all reported malignancies in the digestive tract was 54.49 per 100,000 inhabitants [4]. In addition, the incidence of malignant siNETs amounted to 0.29 per 100,000 inhabitants. Of all malignant tumors of the small intestine, $51.79 \%$ were of neuroendocrine origin [4].

\section{Grading}

Based on the proliferation characteristics linked with more or less malignant behavior, siNETs are for the most part slowly proliferating G1 (37\%) and G2 (63\%) tumors [4]. However, the vast majority of siNETs (48.4\%) presents in stage IV when diagnosed, emphasizing the generally malignant course of all NETs [4].

\section{Indication for Surgery}

The majority of siNETs is found incidentally when examinations for unspecific abdominal symptoms are performed, such as

\section{KARGER}

() 2017 S. Karger GmbH, Freiburg 
Table 1. TNM classification of neuroendocrine tumors of the small intestine

\begin{tabular}{ll}
\hline T- Primary tumor $^{a}$ \\
$\mathrm{x}$ & Primary tumor cannot be assessed \\
0 & No evidence of primary tumor \\
1 & Tumor invades lamina propria or submucosa, size $\leq 1 \mathrm{~cm}$ \\
2 & Tumor invades muscularis propria or size $>1 \mathrm{~cm}$ \\
3 & Tumor invades subserosa without perforation of the serosa \\
4 & $\begin{array}{l}\text { Tumor perforates the visceral peritoneum (serosa) or invades other } \\
\text { organs/neighboring structures }\end{array}$
\end{tabular}

$N$ - Regional lymph node metastasis

$\mathrm{x} \quad$ Regional lymph nodes cannot be assessed

$0 \quad$ No regional lymph node metastasis

1 Lymph node metastasis in up to 12 regional lymph nodes

2 Lymph node metastasis in more than 12 regional lymph nodes or bulks of lymph nodes in the mesenterium (size $>2 \mathrm{~cm}$ )

\section{$M$ - Distant metastasis \\ $\mathrm{x} \quad$ Distant metastasis cannot be assessed \\ $0 \quad$ No distant metastasis \\ 1 Distant metastasis \\ 1a Hepatic metastasis only \\ $1 \mathrm{~b}$ Extrahepatic metastasis only \\ 1c Hepatic and extrahepatic metastasis}

${ }^{\mathrm{a}}$ For any $\mathrm{T}$, add $(\mathrm{m})$ for multiple tumors.

ultrasound, computed tomography, or ileocolonoscopy. Sometimes, siNETs are found incidentally during emergency abdominal surgery due to bowel obstruction (18\% [5]) induced by a primary tumor and/or by extended lymph node metastasis.

Explorations of primaries in incidentally diagnosed liver metastasis sometimes document the NETs.

\section{TNM Classification/Staging of siNETs}

For TNM classification/staging of NETs, criteria summarized by the European Neuroendocrine Tumor Society (ENETS) may be used $[6,7]$. In table 1 and 2, however, the recently published TNM criteria are applied to enable a better understanding of the treatment recommendations [8].

As recently shown [4], 5 (16.1\%) of 31 patients presented with stage I-IIIa (i.e. 'localized') disease and 11 (35.5\%) with stage IIIb (i.e. 'regional') disease, while $15(48.4 \%)$ patients presented with stage IV (i.e. 'distant') disease.

\section{Surgical Treatment}

In general, resection is recommended for localized and regional disease as well as in the setting of impending obstruction and should still be considered for patients with advanced disease. Functional staging using somatostatin receptor scintigraphy is recommended to differentiate localized and regional from distant disease. The results may help in planning surgery and its extent.
Table 2. TNM staging of neuroendocrine tumors of the small intestine

\begin{tabular}{|c|c|c|c|c|c|}
\hline \multirow{2}{*}{$\frac{\text { Stage }}{0}$} & \multicolumn{3}{|l|}{ TNM } & \multirow[t]{2}{*}{$\mathrm{n}(\%)$} & \multirow{2}{*}{$\begin{array}{l}\text { Disease } \\
\end{array}$} \\
\hline & \multicolumn{3}{|c|}{ (Stage 0: ENETS only) } & & \\
\hline I & $\mathrm{T} 1$ & No & M0 & $2(6.5)$ & \multirow{4}{*}{ localized } \\
\hline II & & & & & \\
\hline $\mathrm{a}$ & $\mathrm{T} 2$ & No & M0 & $1(3.2)$ & \\
\hline $\mathrm{b}$ & $\mathrm{T} 3$ & No & M0 & $2(6.5)$ & \\
\hline \multicolumn{6}{|l|}{ III } \\
\hline a & $\mathrm{T} 4$ & No & M0 & 0 & 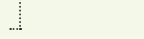 \\
\hline $\mathrm{b}$ & any $\mathrm{T}$ & $\mathrm{N} 1, \mathrm{~N} 2$ & M0 & $11(35.5)$ & regional \\
\hline IV & any $\mathrm{T}$ & any $\mathrm{N}$ & M1, M1a-c & $15(48.4)$ & distant \\
\hline
\end{tabular}

\section{Stage I-III Disease}

All patients are considered as potential candidates for curative surgery of the primary tumor and regional lymph node ( $\mathrm{pN} 1$; selected patients with $\mathrm{pN} 2$ ) metastasis.

At the beginning of the surgical procedure, the small bowel needs to be palpated in its full length to detect or exclude multifocal disease. Any surgical procedure should follow the principles of oncological surgery. The surgical strategy is defined based on the site(s) of the tumor(s). If located in the jejunum or middle ileum, a segment resection is performed. An ileocecal resection or right hemicolectomy may be indicated in tumors located in the distal ileum near the ileocecal valve.

Mesenteric lymph node metastases are invariably involved. Therefore, the recently published TNM classification subdivides patients with pN1 and pN2 lymph node involvement [8].

Bowel resection is always to be performed in combination with meticulous lymph node dissection. Systematic lymphadenectomy may prevent local symptomatology due to obstruction or occlusion of blood supply to the intestine resulting in bowel ischemia. Lymph node metastases may also have a significant impact on the patients' prognosis.

Ohrvall et al. [9] have proposed a currently used surgical 'lymph node staging classification' by evaluating the operability of primary and mesenteric lymph node metastasis. 'Lymph node involvement stage I' consists of tumors located close to the intestine, while stage II of tumors involves arterial branches near their origin in the mesenteric artery. Stage III of lymph node tumors extends along (without encircling) the superior mesenteric artery trunk, and stage IV of lymph node metastases extends retroperitoneally, behind or above the pancreas, or grows around the mesenteric artery and involves the origin of proximal jejunal arteries on the left side of the superior mesenteric artery [10]. The more proximally the lymph node tumor bulk shows mesenteric involvement, the more technically challenging is the approach. The technique of meticulous mesenteric vascular dissection and decompression is sometimes difficult and time-consuming. Surgical dissection of nodes involving the root of the superior mesenteric vessels presents a special challenge and requires an experienced 


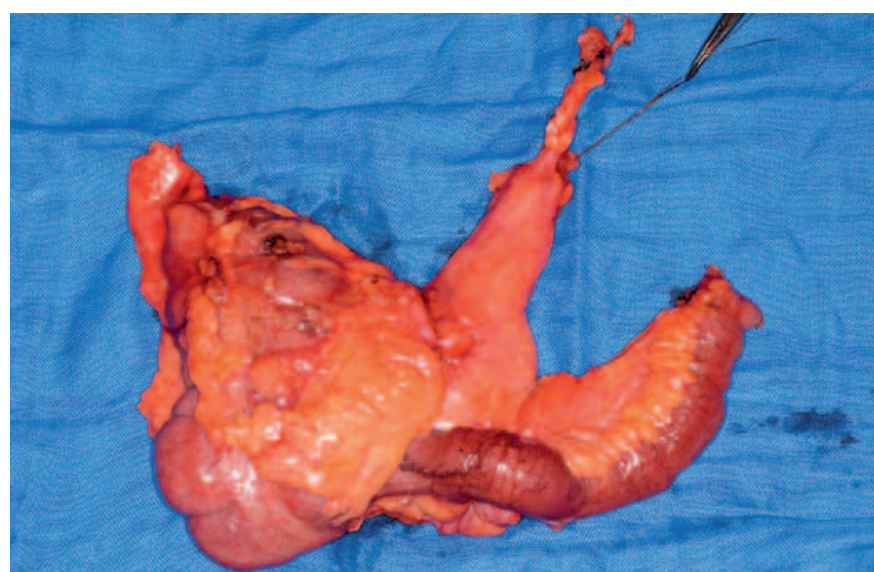

Fig. 1. The neuroendocrine tumor of the small intestine is located near the ileocecal valve; therefore, an ileocecal resection with lymph node dissection along the vascular axis was performed.

surgeon. Obliterative processes with considerable variation in the degree and rate of progression of 'desmoplasia' (i.e. the result of coordinated changes in several stromal cells under the control of a single gene product, CD36, whose repression leads to a decrease in fat accumulation and an increase in matrix deposition [11]) may cause vascular encasement and impede complete and, at times, palliative resection of the central mesenteric lymph node tumor, resulting in vascular ischemia and intestinal obstruction. The distal arterial (and venous) branches (ileocolic artery, right colic artery, ileal or occasionally jejunal arteries) are sometimes to be divided, depending on tumor mass extension (fig. 1). Care has to be taken to preserve important vascular collaterals and arcades along the intestine in order to maintain artery supply to the remaining intestine. Every effort has to be made to preserve sufficient bowel length to preclude short bowel syndrome (fig. 2). However, postoperative malabsorption and/or bile salt-induced diarrhea has been observed due to the resection of more or less extensive parts of the distal small intestine, and particularly the terminal ileum.

Prospective studies have not been performed to assess the efficacy of surgical treatment strategies for neuroendocrine neoplasms of the small intestine. However, some retrospective studies have demonstrated that curative as well as palliative resection of the primary tumor may improve the patients' prognosis and quality of life.

\section{Laparoscopic Approach}

The level of evidence for the role of laparoscopic surgery for siNETs is low. There is a lack of randomized trials. Only few singlecenter studies have described small numbers of patients after laparoscopic resection of primary siNETs (see also [12]). Laparoscopic techniques are feasible and safe and are to be favored due to the general advantages of a laparoscopic surgical approach. However, the potential benefits of minimally invasive surgery are to be weighed against the risk of incomplete (i.e. non-curative) tumor resection (especially in the event of multiple small siNETs).

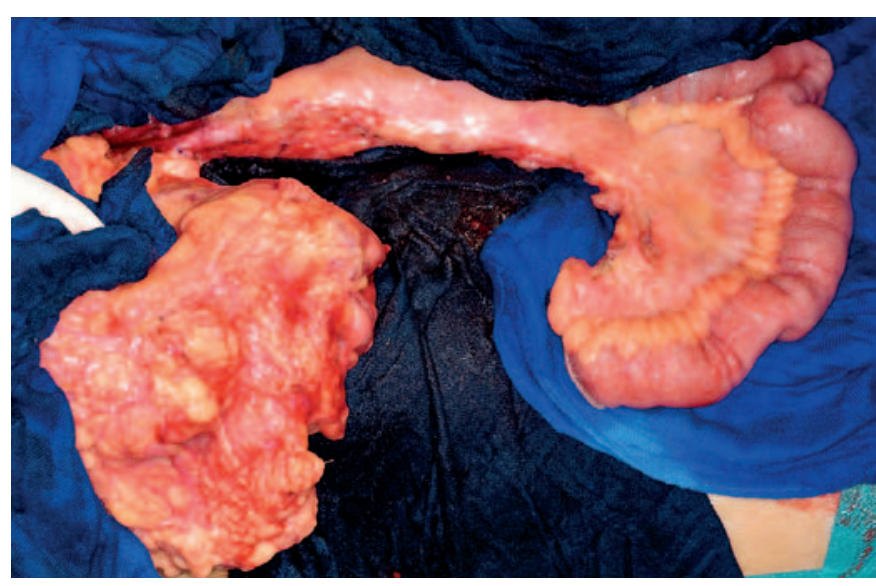

Fig. 2. Care has to be taken to preserve important vascular collaterals and arcades along the intestine to maintain artery supply to the remaining intestine and to preserve sufficient bowel length to preclude short bowel syndrome.

\section{Stage IV Disease - M1a-c}

The pros and cons of surgery of the primary tumor and lymph node metastasis should be carefully discussed in stage IV disease [12].

A small group of patients (less than 10\% [4]) presents with M1a and one single liver metastasis. Following oncology guidelines, this offers the chance of curative treatment for both primary (and the involved lymph nodes) and liver metastases.

In patients with symptoms due to small intestinal obstruction, occlusion, or tumor bleeding, palliative resection of the primary tumor may be mandatory to prevent clinical deterioration or death. In order to avoid local vessel occlusion with ischemic bowel complications by intra-abdominal fibrosis, mesenteric lymph node metastases should be removed as completely as possible.

Palliative (prophylactic) resection of the primary tumor in metastatic disease (M1a) has recently been analyzed in a systematic literature review by Capurso et al. [13]. The available retrospective data suggest a possible benefit of resection of primary siNETs even in patients with unresectable liver metastases.

At first presentation, approximately $30 \%$ of patients with siNETs have peritoneal carcinomatosis (PC). Solitary or multiple PC may occur, frequently accompanied by lymph nodes with (M1c) or without (M1b) liver metastases. The surgical management and the impact of PC on the prognosis of siNETs was recently summarized [14]. Cytoreductive surgery for PC from NETs is feasible in most of the patients and seems to increase survival rates. Aggressive surgical management seems justified for subsets of NET-related PC but requires careful selection of the candidates most likely to benefit. Cytoreductive surgery prolongs survival, especially in M1b, when the peritoneal lesions are completely resected. Too little is known about the benefit of additional hyperthermic intraperitoneal chemotherapy for NET-derived PC. If it offers an advantage, it has to be counterbalanced by its high morbidity. 


\section{Preoperative Evaluation and Preparation}

The majority of patients present with non-specific symptoms, i.e. diffuse abdominal pain (33\%) and melena (15\%) [5]. The typical 'carcinoid syndrome', including flushes (9\%) and/or diarrhea (21\%) [5], and less frequently emesis and bronchoconstriction, is caused by endogenous secretion of mainly serotonin and kallikrein and is overall rare. These clinical symptoms necessitate a special preoperative preparation of the patient.

In patients with suspected carcinoid syndrome, it is recommended that surgery is performed under somatostatin analogs (SSAs) which are initiated preoperatively and applied intravenously during surgery to prevent or treat any carcinoid crisis.

Serotonin and other cytokines released from tumor cells may induce fibrosis, leading to carcinoid heart disease and abdominal fibrotic reactions [15].

Therefore, a careful evaluation of cardiac function is necessary preoperatively.

Although patients with distant metastases may be asymptomatic $[5,12]$, heart failure due to carcinoid heart disease (Hedinger syn- drome) may be concomitant but is rarely the pivotal symptom of the disease $[6,16]$.

\section{Cholecystectomy}

There is an ongoing debate as to whether routine additional cholecystectomy is prophylactically required in non-stone carriers or in asymptomatic stone carriers. The incidence of gallstone-related complications in patients with siNETs appears to be higher than among the general population [17]. Therefore, prophylactic cholecystectomy is liberally recommended during laparotomy in patients with local, regional or distant metastatic disease, as all patients are candidates for long-term treatment with SSAs in an effort to decrease symptoms and/or to prolong progression-free survival $[18,19]$.

\section{Disclosure Statement}

The authors have no conflict of interest to declare. This paper is not based on a previous communication to a society or a meeting.

\section{References}

1 Lubarsch O: Ueber den primären Krebs des Ileum nebst Bemerkungen über das gleichzeitige Vorkommen von Krebs und Tuberculose. Virchows Arch Pathol Anat 1888;111:280-317

2 Oberndorfer S: Karzinoide Tumoren des Duenndarms. Frankf Z Pathol 1907;1:426-429.

3 Bosman FT: WHO classification of tumours of the digestive system. Lyon, IARC Press, 2010.

4 Niederle MB, Hackl M, Kaserer K, Niederle B: Gastroenteropancreatic neuroendocrine tumours: the current incidence and staging based on the WHO and European Neuroendocrine Tumour Society classification: an analysis based on prospectively collected parameters. Endocr Relat Cancer 2010;17:909-918.

5 Niederle MB, Niederle B: Diagnosis and treatment of gastroenteropancreatic neuroendocrine tumors: current data on a prospectively collected, retrospectively analyzed clinical multicenter investigation. Oncologist 2011;16:602-613.

6 Rindi G, Klöppel G, Couvelard A, Komminoth P, Körner M, Lopes JM, McNicol AM, Nilsson O, Perren A, Scarpa A, Scoazec JY, Wiedenmann B: TNM staging of midgut and hindgut (neuro) endocrine tumors: a consensus proposal including a grading system. Virchows Arch 2007;451:757-762.

7 Rindi G, Klöppel G, Alhman H, Caplin M, Couvelard A, de Herder WW, Erikssson B, Falchetti A, Falcon M, Komminoth P, Körner M, Lopes JM, McNicol AM Nilsson O, Perren A, Scarpa A, Scoazec JY, Wiedenmann B: TNM staging of foregut (neuro)endocrine tumors: a consensus proposal including a grading system. Virchows Arch 2006;449:395-401.
8 Brierley JD, Gospodarowicz MK, Wittekind C (eds): TNM Classification of Malignant Tumours, ed 8. Hoboken, NJ, Wiley-Blackwell, 2016, pp 133-134.

9 Ohrvall U, Eriksson B, Juhlin C, Karacagil S, Rastad J, Hellman P, Akerström G: Method for dissection of mesenteric metastases in mid-gut carcinoid tumors. World J Surg 2000;24:1402-1408.

10 Boudreaux JP, Klimstra DS, Hassan MM, Woltering EA, Jensen RT, Goldsmith SJ, Nutting C, Bushnell DL, Caplin ME, Yao JC; North American Neuroendocrine Tumor Society (NANETS): The NANETS consensus guideline for the diagnosis and management of neuroendocrine tumors: well-differentiated neuroendocrine tumors of the Jejunum, Ileum, Appendix, and Cecum. Pancreas 2010:39:753-766.

11 DeClerk YA: Desmoplasia: a response or a niche? Cancer Discov 2012:2:772-774.

12 Niederle B, Pape UF, Costa F, Gross D, Kelestimur F, Knigge U, Öberg K, Pavel M, Perren A, Toumpanakis C, O'Connor J, O'Toole D, Krenning E, Reed N, Kianmanesh R; Vienna Consensus Conference participants: ENETS Consensus Guidelines Update for neuroendocrine neoplasms of the jejunum and ileum. Neuroendocrinology 2016;103:125-138.

13 Capurso G, Rinzivillo M, Bettini R, Boninsegna L, Delle Fave G, Falconi M: Systematic review of resection of primary midgut carcinoid tumour in patients with unresectable liver metastases. Br J Surg 2012:99; 1480-1486.
14 de Mestier L, Lardière-Deguelte S, Brixi H, O’Toole D, Ruszniewski P, Cadiot G, Kianmanesh R: Updating the surgical management of peritoneal carcinomatosis in patients with neuroendocrine tumors. Neuroendocrinology 2015:101:105-111.

15 Daskalakis K, Karakatsanis A, Stålberg P, Norlén O, Hellman P: Clinical signs of fibrosis in small intestinal neuroendocrine tumours. Br J Surg 2017;104:69-75.

16 Luis SA, Pellikka PA: Carcinoid heart disease: diagnosis and management. Best Pract Res Clin Endocrinol Metab 2016;30:149-158.

17 Norlén O, Hessman O, Stålberg P, Akerström G, Hellman P: Prophylactic cholecystectomy in midgut carcinoid patients. World J Surg 2010;34:1361-1367.

18 Caplin ME, Pavel M, Ćwikła JB, Phan AT, Raderer M, Sedláčková E, Cadiot G, Wolin EM, Capdevila J, Wall L, Rindi G, Langley A, Martinez S, Blumberg J, Ruszniewski P; CLARINET Investigators: Lanreotide in metastatic enteropancreatic neuroendocrine tumors. N Engl J Med 2014;371:224-233.

19 Caplin ME, Pavel M, Ćwikła JB, Phan AT, Raderer M, Sedláčková E, Cadiot G, Wolin EM, Capdevila J, Wall L, Rindi G, Langley A, Martinez S, Gomez-Panzani E, Ruszniewski P; CLARINET Investigators: Anti-tumour effects of lanreotide for pancreatic and intestinal neuroendocrine tumours: the CLARINET open-label extension study. Endocr Relat Cancer 2016;23:191199. 